\title{
Criminologie
}

\section{Sécurité privée au Québec, un marché en évolution ?}

\section{Fabienne Degailler}

Volume 31, numéro 2, automne 1998

La sécurité privée

URI : https://id.erudit.org/iderudit/017418ar

DOI : https://doi.org/10.7202/017418ar

Aller au sommaire du numéro

Éditeur(s)

Les Presses de l'Université de Montréal

ISSN

0316-0041 (imprimé)

1492-1367 (numérique)

Découvrir la revue

Citer cet article

Degailler, F. (1998). Sécurité privée au Québec, un marché en évolution ? Criminologie, 31(2), 47-67. https://doi.org/10.7202/017418ar

\section{Résumé de l'article}

The majority of studies centering on the evolution of private security use a count of agents, agencies, or both as a focus of analysis. This article is concerned with the possible biases and validity problems related to such indicators. An economic framework is, in turn, proposed to study the market and evolution of private security. Trends observed include a decline in the market for guarding and an increase in the private investigation market, with the later, however, continuing to represent only a small share of the overall private security market. It is argued that one cannot conclude on the decline in the overall market for private security because data on expanding and new technologies in the field are lacking. 
SÉCURITÉ PRIVÉE AU QUÉBEC, UN MARCHÉ EN ÉVOLUTION ?'

Fabienne Degailler ${ }^{2}$

The majority of studies centering on the evolution of private security use a count of agents, agencies, or both as a focus of analysis. This article is concerned with the possible biases and validity problems related to such indicators. An economic framework is, in turn, proposed to study the market and evolution of private security. Trends observed include a decline in the market for guarding and an increase in the private investigation market, with the later, however, continuing to represent only a small share of the overall private security market. It is argued that one cannot conclude on the decline in the overall market for private security because data on expanding and new technologies in the field are lacking.

\section{INTRODUCTION}

Avant le $\mathrm{XIX}^{e}$ siècle, la sécurité privée représente le seul moyen d'assurer la protection de l'individu. Avec l'avènement de l'État-nation, l'ordre des choses est renversé : l'État se réserve le monopole du contrôle social formel, notamment par la mise en force d'une police publique universelle. La seconde guerre mondiale marque un autre renversement de situation avec la croissance de nouveaux moyens de protection, technologiques, mis sur le marché par des entreprises privées. À l'heure actuelle, et depuis un certain temps déjà, on ne met plus en doute l'expansion considérée comme étant phénoménale des services de sécurité privée : c'est la conclusion à laquelle en arrive l'ensemble des auteurs traitant de l'évolution et de la diversité des services offerts en matière de sécurité privée (Farnell et Shearing, 1977 ; Spitzer et Scull, 1977 ; Shearing et Stenning, 1983, 1987 ; Shearing, 1984 ; Ocqueteau, 1986 ; Fourcaudot, 1988 ; McCrie, 1988 ; Cunningham, Stauschs et Van Meter, 1990 ; Dedecker, 1991 ; Nogala, 1996).

1. Cette étude a été réalisée grâce à une subvention du Conseil de recherches en sciences humaines (CRSH, volet petites subventions) et à une subvention du Fond concerté d'aide à la recherche (FCAR, volet jeunes chercheurs). L'auteure a aussi bénéficié d'une bourse d'études octroyée par le Centre international de criminologie comparée.

2. M. Sc. en criminologie. L'essentiel des données traitées dans cet article sont issues du mémoire de maîtrise de l'auteure, intitulé « Étude de marché des agences de sécurité à contrat, à Montréal et au Québec (1986-1996) », réalisé sous la direction de Marie-Marthe Cousineau, professeure à l’École de criminologie de l'Université de Montréal. 
Selon les chercheurs qui traitent de la question, la sécurité privée est considérée soit comme un substitut ou un compétiteur, soit comme un complément à la police publique ; mais dans tous les cas la conclusion reste la même : il y a extension et intensification du contrôle exercé sur la population par la mise en place de services de sécurité privée de plus en plus nombreux et variés.

L'extension et l'intensification du contrôle dans toutes les sphères de la vie privée des individus préoccupent certains auteurs. Marx (1988), notamment, s'inquiète des restrictions pernicieuses des libertés individuelles associées à la prolifération des activités des agences et agents de sécurité privée. L'auteur crée ainsi la phobie d'une société de type totalitaire où chacun se verrait contrôlé dans ses moindres faits et gestes. Clarke (1995), pour sa part, pense que ces craintes sont injustifiées car, dit-il, un fort attachement à la liberté individuelle et la réalité des coûts économiques limiteront strictement les niveaux de surveillance et de sécurité. De fait, de l'avis de Clarke (1995: 11): «l'omniprésence de la sécurité semble moins inquiétante que la non mise en place de mesures de prévention sensées et raisonnables, qui sont rejetées au nom de ce que certains considèrent comme les libertés et les droits des individus ».

D'un certain point de vue, on constate, en effet, que les gains liés à l'implantation de moyens de protection modernes, telles les mesures technologiques, ou plus traditionnels, tels les agents de sécuritê, sont indéniables, notamment en regard de la prévention (Cusson, 1992 ; Clarke, 1995) et de la réduction des coûts liés au contrôle pénal d'événements criminalisables (Cousineau et Normandeau, 1993). Ces coûts sont réels et mesurables (quantifiables), mais ils sont aussi de nature psychologique, cette fois inestimables, et touchent non seulement l'individu mais aussi, dans bien des cas, la société dans son ensemble. C'est pourquoi, diront Shearing et Stenning (1983), la demande de sécurité ne vise pas seulement une diminution de la probabilité d'être victime, mais sert également à augmenter le sentiment de sécurité. L'utilisation de moyens de protection apparaît dès lors comme un complément à d'autres mesures destinées à enrayer le processus de victimisation et les conséquences qui en découlent, et à augmenter ainsi le bien-être social (Lipman et McGraw, 1988).

Nous ne nous donnons pas pour mission ici de prendre position dans le débat des torts et des bienfaits de la sécurité privée. D'autres auteurs, dans ce numéro et ailleurs, en discutent abondamment et très justement. Notre propos consiste plutôt à se demander s'il y a lieu de s'inquiéter, données à l'appui, de l'évolution, que l'on dit sans cesse croissante, de la sécurité privée. Nous trouvions toutefois important de rappeler d'entrée de jeu l'existence de ce débat, qui à la fois découle et se nourrit des études portant sur l'évolution de la sécurité privée. 


\section{MÉTHODOLOGIE}

On peut voir la sécurité soit comme un bien, soit comme un droit. Il existe deux courants de pensée sociologiques soutenant ces positions a priori contradictoires (Ocqueteau, 1992). Les sociologies normatives d'inspiration durkheimienne, sur une base idéologique et historique, posent la sécurité comme un droit. Les sociologies néoréalistes, s'appuyant quant à elles sur la rationalité de l'homme - voulant que pour chacune de ses actions celui-ci fasse une analyse côtts-bénéfices qui détermine ses choix -, considèrent plutôt la sécurité comme un bien. Pour notre part, nous concevons que l'un n'exclut pas l'autre : tout dépend du plan sur lequel on se place. D'un point de vue macro-sociologique, chacun a le droit de prétendre à un minimum de sécurité (Nogala (1996) parle de mission sociale de sécurité); et d'un point de vue macro-sociologique, chacun a le pouvoir de s'organiser afin d'augmenter sa sécurité. Étant donné que notre analyse porte sur le comportement individuel dans la recherche de sécurité, nous traitons de la sécurité comme d'un bien, ou plutôt d'un service, en considérant l'évolution contemporaine de la fourniture d'un tel service.

Le secteur d'activité de la sécurité privée touché par notre étude est celui des agences de sécurité à contrat fournissant les services d'agents de gardiennage dans le but de protéger des biens et des personnes. Bien que les agences de sécurité à contrat ne constituent qu'une facette de la sécurité privée, elles n'en occupent pas moins, au Québec, la part la plus importante.

L'hypothèse que nous tentons de vérifier s'énonce comme suit: «le marché de la sécurité privée dont le secteur d'activité est la protection des biens et des personnes par des agents de sécurité (gardiennage privé) n'est plus en croissance. Il y a substitution par les moyens de protection technologiques, ce qui amène une modification du contrôle social $»$. Clairement, cette hypothèse de base se découpe en deux morceaux. Or, si nous avons pu, avec moult difficultés, répondre à la première partie de la proposition et montrer qu'effectivement le marché du gardiennage privé, au Québec, non seulement stagne mais régresse, la deuxième partie de l'énoncé, portant sur la substitution par les moyens de protection technologiques et, par conséquent, sur la transformation du contrôle social, reste tout entière à explorer. Mais il s'agirait là d'une toute autre étude dont la faisabilité reste encore à établir'. Il s'agira

3. Les séries de données nécessaires pour analyser l'effet de substitution entre le gardiennage et la technologie sont : 1) les prix et, 2) les chiffres d'affaires pour les deux secteurs d'activités. Or, à l'heure actuelle, les deux séries sont agrégêes, et il ne semble pas que l'on puisse les obtenir autrement. Dès lors, une cueillette de données imposante doit être réalisée, exigeant des investissements considérables de temps et d'argent dont nous ne disposions pas. 
donc, dans le cadre de cet article, de rendre compte des moyens utilisés qui nous ont amenée à conclure au recul du secteur gardiennage de la sécurité privée.

La méthodologie utilisée aux fins de notre étude est purement quantitative. Plusieurs sources de données ont été mises à contribution avec plus ou moins de succès. C'est finalement dans le Répertoire des agences de sécurité et d'investigation de 1996, et grâce aux données fournies par le Comité paritaire ${ }^{4}$ chargé de surveiller l'application du Décret sur les agents de sécurité que nos analyses ont pu être menées, principalement à partir d'analyses de régression.

\section{LES INDICATEURS DE L'ÉVOLUTION DE LA SÉCURITÉ PRIVÉE}

Pour mesurer la croissance de la sécurité privée, deux indicateurs sont couramment utilisés dans les études, notamment criminologiques : l'évolution du nombre d'agences et l'évolution du nombre d'agents de sécurité. C'est principalement en regard de ce dernier indicateur que les chercheurs concluent que le marché de la sécurité privée a supplanté en taille la police publique. Notre propos consiste d'abord à montrer que ces deux indicateurs comportent de tels biais que leur interprétation laisse à désirer. En second lieu, en se basant plutôt sur une analyse de la structure de marché, un indicateur moins bien connu des criminologues, nous constatons que, contrairement à ce qu'indiquent les deux premiers indicateurs, à tout le moins depuis 1989, au Québec, le marché de la sécurité privée à contrat est en régression.

\section{Évolution du nombre d'agences de sécurité et d'investigation au Québec}

Au Québec, selon le règlement d'application de la Loi sur les agences d'investigation et de sécurité (L.R.Q., c A-8, r.1), moyennant un certain nombre de conditions, n'importe quel citoyen canadien solvable, âgé au moins de 21 ans et possédant une expérience pertinente d'au moins cinq ans dans le domaine de l'investigation ou de la sécurité, à quelque titre que ce soit, ou dans un corps policier reconnu, et disposant d'un bureau d'affaires au Québec, peut obtenir un permis d'agence de sécurité privée, lequel est renouvelable au 31 mars de chaque année. Il existe trois types de permis : de sécurité, d'investigation ou mixte (sécurité et investigation). Pour chacun de ces permis, le droit à payer est différent, variant, en 1996, de $1042 \$$ à $3127 \$$, et est assorti de cautionnements variant entre $5000 \$$ et $10000 \$$.

4. Le Comité paritaire se compose d'un nombre égal de représentants, d'employeurs et de salariés du secteur de la sécurité privée à contrat. 
Un répertoire publié par le ministère de la Sécurité publique fournit chaque année un certain nombre de données sur les agences de sécurité et d'investigation licenciées. Tout ce qui concerne le secteur de la sécurité interne échappe à ce recensement.

Étant donné que la date de recensement n'est pas la même chaque année, un scatter-plot a été utilisé pour représenter graphiquement les données tirées de cette source, cela pour éviter d'être induit en erreur par les distances variables entre les dates d'inscriptions (graphique 1).

\section{Graphique 1}

Évolution du nombre d'agences de sécurité, d'investigation et mixtes. au Québec de 1986 à 1996, à date fixe

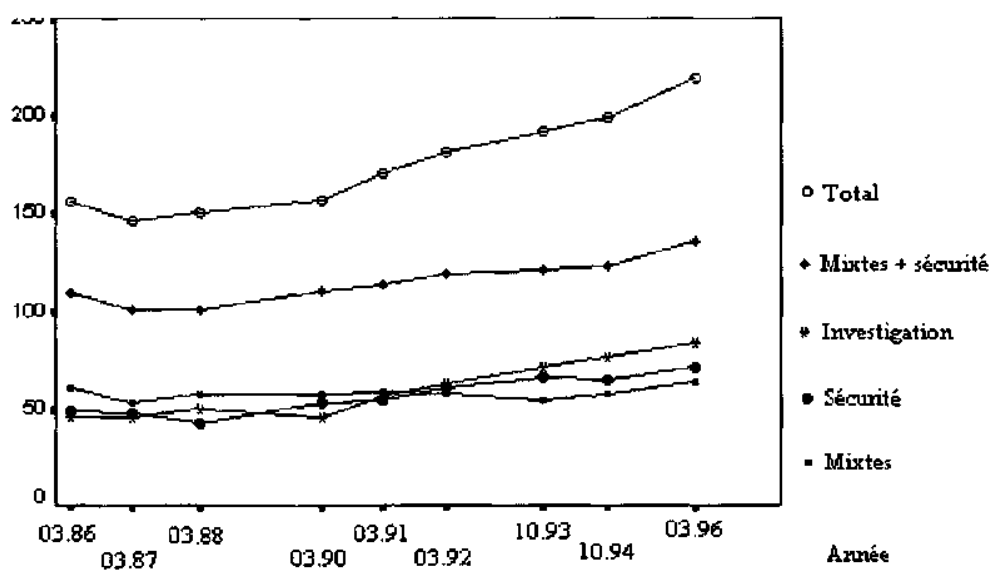

En observant le graphique 1, on constate que :

- toutes les séries présentent une tendance à la hausse, exception faite des agences mixtes pour lesquelles la série stagne sur toute la période ;

- la croissance du nombre d'agences d'investigation est supérieure aux autres ;

- la phase de stagnation observée à compter de 1986 par Fourcaudot (1988) se poursuit jusqu'en 1988, avant que ne reprenne apparemment une phase de croissance.

Pour vérifier s'il y a croissance ou stagnation du nombre de permis d'agences de sécurité privée durant la période étudiée, on teste l'hypothèse unilatérale $\mathrm{H}_{\mathrm{A}}: \mathrm{b}>0$ contre $\mathrm{H}_{0}: \mathrm{b}=0$, b représentant le coefficient de 
croissance. Le seuil critique de la statistique de Student à $5 \%$, pour 9 observations est $\mathrm{t}_{(0.05,7)}=1,90$. Les résultats des différentes régressions, selon le type d'agences (sécurité, investigation ou mixte), sur une constante et une tendance sont présentés au tableau 1. Ceux-ci montrent que seul le nombre d'agences mixtes n'augmente pas significativement. Les tendances appréhendées à partir des données de Fourcaudot (1988) ne se confirment pas, car le secteur des agences de sécurité croît. On doit donc en conclure que la phase de stagnation amorcée au début des années 1980, pour l'ensemble des agences, n'était que passagère, laissant présager qu'il s'agissait plutôt d'une phase de ralentissement durant une période de croissance.

Tableau 1

Vérification de tendance concernant l'évolution des différents secteurs d'activités du marché contractuel de la sécurité (1986-1996)

\begin{tabular}{|c|c|c|c|}
\hline Variables & Constante & Tendance & R-carré \\
\hline Mixtes & $\begin{array}{c}56,75 \\
(28,40)\end{array}$ & $\begin{array}{c}0,87 \\
(0,41)\end{array}$ & 0,10 \\
\hline Sécurité & $\begin{array}{c}43,33 \\
(19,71)\end{array}$ & $\begin{array}{c}0,22 \\
(7,29)^{*}\end{array}$ & 0,88 \\
\hline $\begin{array}{c}\text { Mixtes et } \\
\text { sécurité }\end{array}$ & $\begin{array}{c}100,08 \\
(31,66)\end{array}$ & $\begin{array}{c}0,24 \\
(5,62)^{*}\end{array}$ & 0,82 \\
\hline Invest & $\begin{array}{c}39,48 \\
(12,44)\end{array}$ & $\begin{array}{c}0,33 \\
(7,65)^{*}\end{array}$ & 0,89 \\
\hline Total & $\begin{array}{c}139,56 \\
(26,15)\end{array}$ & $\begin{array}{c}0,57 \\
(7,88)^{*}\end{array}$ & 0,90 \\
\hline
\end{tabular}

$* \mathrm{p}<0,05$

Le premier nombre est la valeur du coefficient, le nombre entre parenthèses esl la valeur du t-stat.

Étant donné la confirmation de croissance des sous-secteurs du gardiennage et de l'investigation, ainsi que du nombre total des agences, on s'intéresse maintenant à l'amplitude de cette croissance entre 1986 et 1996. Afin d'éliminer l'effet de la phase de stagnation observée en début de période, la croissance est calculée à partir de 1988. L'amplitude de la croissance des entreprises mixtes n'est pas étudiée étant donné qu'elle n'apparaît pas significative. Une autre analyse, que nous ne présenterons pas ici (voir à ce sujet Degailler, 1997), nous apprend que le secteur d'activité qui croît le plus est le secteur de l'investigation. Cependant, depuis 1988, la croissance du nombre 
d'agences fournissant des services de gardiennage ou d'investigation est quasiment équivalente. La croissance totale du nombre d'agences de sécurité paraît moindre, ce qui est dû à la stagnation de l'évolution des agences de sécurité mixtes.

En définitive, considérant le nombre d'agences de sécurité comme indicateur de croissance, on constate que :

- pour l'ensemble du Québec, globalement, le marché de la sécurité à contrat est en hausse, malgré une phase de déclin au cours des années 1980 ;

- le secteur d'investigation crôit davantage que celui du gardiennage ;

- après une phase de stagnation jusqu'en 1987, le secteur du gardiennage recommence à croître ;

- le développement des entreprises mixtes stagne sur toute la période.

Cependant, cette méthode d'analyse comporte plusieurs biais. Ainsi :

- seules les succursales sont comptabilisées et non pas les filiales. Par conséquent, il y a sous-évaluation systématique du nombre réel des commerces fournissant des services de gardiennage et d'investigation privés, ou les deux, sur une base contractuelle. Le nombre de filiales n'étant pas disponible, il est impossible de préciser les séries précédentes en conséquence ;

- cet indicateur ne donne aucune idée de la taille des entreprises. Cela peut créer des distorsions dans l'analyse. Par exemple, si une entreprise se scindait en deux, toute chose étant égale par ailleurs, nous conclurions à une augmentation du marché, alors qu'il n'en est rien. Une deuxième possibilité est que, malgré l'augmentation du nombre d'entreprises, nous puissions observer, parallèlement, une forte baisse du chiffre d'affaires, indiquant par là même que le marchê est en récession;

- un autre problème concerne l'analyse de la stabilité. Il est possible que l'on retrouve un nombre constant d'entreprises sur toute la période observée, mais que chacune ait augmenté son nombre de clients. Par conséquent, en réalité, le marché serait en expansion. L'inverse est aussi vrai ;

- il peut y avoir une grande rotation des entreprises, car celles-ci ont des difficultés à s'implanter sur le marché et font rapidement faillite ;

- et enfin, le fait que le sous-secteur gardiennage paraisse augmenter après une phase de déclin peut conduire à des conclusions erronées. L'évolution du nombre d'agences n'indique pas si l'augmentation est une réponse à une nouvelle forme de demande (à ce moment-là, on a un nouveau produit et, par conséquent, un changement de marché) ou si elle constitue le secteur d'activité principal de l'entreprise (la sécurité est un à-côté) ou si, finalement, on a simplement identifié une phase de ralentissement du marché à l'intérieur d'une période de croissance. 
En conséquence, pris comme seul indicateur, l'évolution du nombre d'entreprises peut conduire à une mauvaise interprétation de l'évolution du marché de la sécurité privée, la liste des points problématiques énoncés cidessus n'étant d'ailleurs pas exhaustive.

Une façon de contourner ces biais consiste, entre autres, à évaluer l'évolution du nombre d'agents de sécurité.

\section{Évolution du nombre d'agents de sécurité et d'investigation au Québec}

Étant donné que l'étude porte sur un marché à forte intensité en maind'œuvre, l'évolution du nombre d'agents de sécurité constitue une méthode d'analyse possible de ce marché.

La Direction des permis de la Sûreté du Québec possède des statistiques concernant le nombre de permis accordés (nouveaux et renouvelés) pour les agents de sécurité et d'investigation. Malheureusement le permis émis étant le même dans les deux cas, aucune distinction ne peut être faite entre ces deux catégories d'agents. Selon Gérard Laurin, enquêteur spécialisé et conseiller en prévention du crime, le nombre actuel d'investigateurs actifs au Québec ne dépasse pas 500 personnes ${ }^{5}$ malgré l'importante augmentation du nombre d'entreprises d'investigation ${ }^{6}$. L'augmentation du nombre d'entreprises d'investigation ne serait en fait qu'illusoire : il s'agirait, soit d'entreprises de sécurité déjà existantes ayant séparé leurs deux secteurs d'activités, soit d'anciens employés d'entreprises de sêcurité travaillant maintenant seuls, à leur compte.

On observe que la croissance phénoménale du nombre d'agents de sécurité (Shearing, 1984 ; Truett, Tillet et Van Meter, 1988) n'apparaît plus présente aujourd'hui (graphique 2). On note aussi que deux chocs ont eu lieu, en 1987 et 1991. Ceux-ci sont, dans les deux cas, en grande partie attribuables à des mouvements de grèves de la Société canadienne des postes. Ces chocs se traduisent par des hausses extraordinaires du nombre total de permis émis, ainsi que du nombre de nouvelles demandes de permis pour l'exercice de fonctions d'agents de sécurité en relation avec des événements précis commandant leur présence pour une période donnée. En dehors de ces deux pics, inhabituels, on note que, sur l'ensemble de la période étudiée, le nombre total de permis émis, ainsi que le nombre de nouvelles demandes de permis enregistrées diminuent significativement.

5. Le nombre obtenu par Fourcaudot, en 1988, est de 440.

6. Pour l'investigation, la croissance annuelle moyenne du nombre d'agences, de 1986 à 1996 , est de $5,63 \%$. 


\section{Graphique 2}

Évolution du nombre d'agents de sécurité et d'investigation au Québec de 1980 à 1995

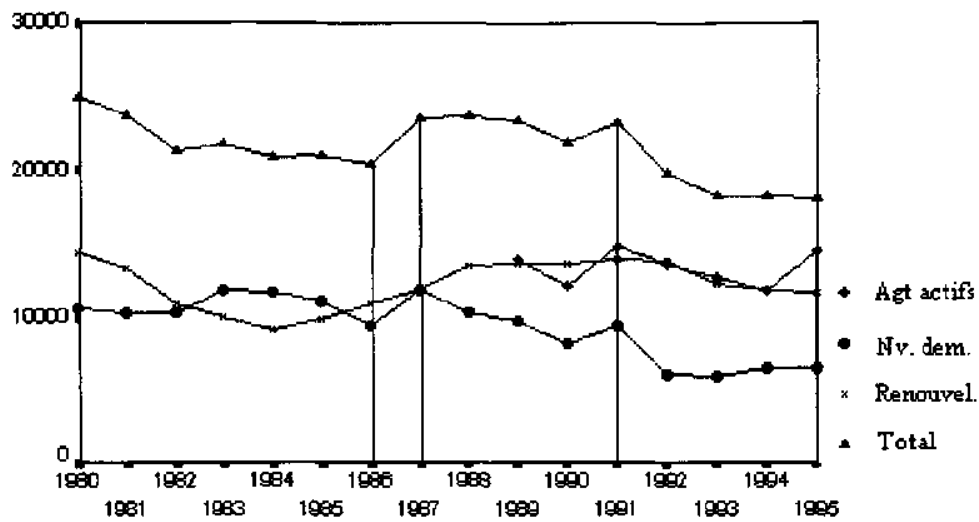

Agt actifs est le nombre total d'agents de sécurité actifs pour l'année considérée ; $\mathrm{Nv}$. dem., le nombre de nouvelles demandes de permis ; Renouvel., le nombre de permis renouvelés ; et Total, le nombre total de permis émis.

Comme le nombre de nouvelles demandes diminue alors que le nombre d'agents actifs et le nombre de renouvellements restent stables, on peut conclure qu'actuellement le roulement de personnel est moins grand qu'au début des années 1980 et que la diminution de personnel s'est surtout faite au détriment des agents temporaires.

Enfin, dans tous les cas, on constate que le nombre d'investigateurs est faible comparativement aux gardiens, ce qui laisse à penser que le comportement de ces derniers n'aurait pas une grande influence sur l'évolution des permis demandés.

Au delà de ces quelques constatations de base, et modulant la fiabilité de celles-ci, plusieurs problèmes se présentent lors de l'analyse de l'évolution du marché des agences de sécurité à contrat à partir de ses effectifs, dont les principaux sont les suivants :

- le premier, et le plus important, est le manque de données disponibles ;

- en outre, la plupart des données disponibles ne sont pas parfaitement fiables car, par exemple, il est connu que les agences transmettent des données erronées au Comité paritaire notamment en ce qui concerne le nombre d'agents qu'elles emploient. N'ayant aucune idée de la proportion que cette distorsion représente, aucune correction ne peut être apportée à l'analyse des effectifs ; 
- tous les agents ne sont pas recensés, en raison du travail au noir, et nous ne savons pas si celui-ci a augmenté ou diminué au fil des ans ;

- un autre problème concerne les agents engagés à temps partiel ou pour des durées limitées. Par exemple, deux agents engagés à demi temps ont exactement le même poids dans l'analyse qu'un agent engagé à temps plein. À cet égard, la prise en compte du taux d'occupation (nombre d'heures effectivement travaillées en regard d'un temps complet qui, pour la sécurité privée, se chiffre à 42 heures par semaine) permet une correction des séries, spécialement pour le cas où ce taux n'est pas stable dans le temps ;

- le point précédent vaut aussi lors de la comparaison entre le nombre d'agents de sécurité et le nombre de policiers. La meilleure façon d'analyser la situation, dans ce cas-ci, est d'utiliser l'évolution du nombre d'heures travaillées.

En définitive, cet indicateur peut facilement être mal interprété et, surtout, il ne peut pas être analysé sans tenir compte des heures travaillées. Par ailleurs, connaissant le nombre d'heures travaillées, cet indicateur ne sert plus à rien, car les fluctuations du marché peuvent dès lors être directement déterminées, comme nous le verrons plus loin.

\section{STRUCTURE DE MARCHÉ}

L'analyse des deux indicateurs précédents pour juger de l'évolution du marché de la sécurité privé est pratique courante en criminologie. Par contre, l'étude de la structure de marché appliquée au marché de la sécurité privée,

- probablement parce qu'elle exige des connaissances économiques - n'a pas encore été faite en criminologie, du moins à notre connaissance.

L'analyse de la structure de marché, dont le cadre théorique tient de l'organisation industrielle et de la théorie des jeux, s'intéresse au comportement des entreprises et des consommateurs, ainsi qu'à la façon dont chacun interagit. Il s'agit donc d'une analyse de type micro-économique.

D'abord, il s'agit de déterminer le type de marché auquel on fait face (en l'occurrence soit un monopole, soit une concurrence parfaite, soit un oligopole). Ensuite, on peut se demander à quel stade du cycle de vie le produit ou service étudié, ici les services contractuels de sécurité privée, se situe.

\section{À quel type de marché correspond le marché de la sécurité privée ?}

Rapidement, on peut conclure qu'il ne s'agit pas d'un marché monopolistique. Le nombre d'entreprises fournissant des services de gardiennage et d'investigation privés au Québec, et le fait que plusieurs d'entre elles soient 
de grande taille, nous force d'entrée de jeu à conclure qu'il ne s'agit pas d'un monopole. Dès lors, on cherche à établir si on se trouve en situation d'oligopole (plusieurs entreprises sont présentes sur le marché mais un petit nombre d'entre elles s'en partage la plus grande part) ou de concurrence parfaite. Pour conclure à une situation de concurrence parfaite, plusieurs conditions doivent être remplies : 1 ) on se trouve en présence d'un grand nombre de vendeurs ou de fournisseurs de service et d'un grand nombre d'acheteurs ; 2) le bien ou le service est homogène, de même que le prix de fourniture en vigueur ; 3) il n'existe pas de barrières à l'entrée (ni à la sortie).

Étant donné que la situation de monopole peut-être éliminée, il s'agit de vérifier si les conditions de la concurrence parfaite sont remplies. Dans le cas contraire, nous nous pencherons sur l'hypothèse de la présence d'un oligopole, une telle structure de marché étant beaucoup plus difficile à établir directement.

\section{Homogénéité du prix et des services}

Le prix des services contractuels de sécurité privée, au Québec, est relativement homogène. 11 existe des variations, mais qui ne sont que de l'ordre de quelques dollars. Cette situation est en grande partie due au Décret sur les agents de sécurité (R.R.Q., 1981, chap. D-2, r.1), qui fixe le salaire horaire des employés de sécurité, faisant en sorte que celui-ci soit, à toutes fins utiles, le même pour toutes les entreprises de sécurité, les employés étant payés au salaire minimum de 10,60 dollars/heure ${ }^{7}$. En moyenne, le prix coûtant (prix de revient) par heure pour un agent de sécurité est de 13 dollars et le taux horaire demandé aux clients varie généralement entre 13 et 15 dollars. Il existe cependant des exceptions pour certaines entreprises utilisant les services $d^{\prime}$ agents subventionnés ${ }^{8}$ : celles-ci paient leurs agents environ huit dollars de l'heure. Mais il s'agit de cas d'exception.

Une autre condition pour qu'un marché soit en concurrence parfaite est l'homogénéité du bien ou des services rendus. Dans le cas des agents de sécurité, il est douteux que le bien soit parfaitement homogène, la qualité des services variant entre les entreprises. Ces différences se jouent de plusieurs façons, bien que les services offerts par les grandes entreprises soient quasiment identiques. Entre autres, des différences se remarquent dans :

- la formation des agents. Certaines entreprises exigent des postulants qu'ils suivent des cours ;

7. Il existe des primes additionnelles selon la formation de l'agent, mais celles-ci demeurent marginales et peu importantes.

8. Via différents programmes gouvernementaux d'employabilité (CSST, PAIE, ...) 
- la façon de traiter le client. De plus en plus, le client est traité en tant que partenaire que l'on consulte à chaque étape décisionnelle. On ne lui impose plus un concept de sécurité, mais on en discute avec lui ;

- la diversification des services de l'entreprise. Par exemple, Pinkerton possède plusieurs branches d'activités incluant un service d'alarmes. Si un client désire plusieurs services de sécurité, cette entreprise peut les lui fournir, alors qu'une petite entreprise devra envoyer le client ailleurs pour les services qu'elle n'offre pas.

\section{Barrières à l'entrée}

Les barrières à l'entrée représentent tout obstacle qui empêche une nouvelle entreprise de s'implanter sur le marché. Elles peuvent être soit créées par le marché, soit imposées par l'État, soit générées par une entreprise ayant une position dominante. Dans une situation de concurrence parfaite, il ne doit y avoir ni barrière à l'entrée, ni à la sortie.

La province de Québec impose un certain nombre de conditions afin d'obtenir un permis d'agence de sécurité. Cependant, ces conditions ne sont pas très contraignantes et le prix demandé n'est pas prohibitif. Il ne s'agit donc pas de barrières réelles.

Le prix des services de sécurité privée à contrat étant homogène dû au Décret sur les agents de sécurité, il ne peut y avoir de guerres des prix et les entreprises ne peuvent, par conséquent, ériger des barrières agressives. La réputation de l'entreprise auprès des clients devient alors primordiale et l'utilisation de la publicité constitue un moyen de l'améliorer. Le port d'un uniforme distinctif devient aussi important, car il permet aux entreprises de se faire (re)connaître auprès des clients potentiels.

En fait, il apparaît que le marché lui-même impose des conditions telles que les petites entreprises ne peuvent soumissionner pour n'importe quel contrat. Le client exigera, par exemple, en particulier s'il s'agit de gros contrats, que l'entreprise ait maintenu un chiffre d'affaires minimum au cours des $\mathrm{x}$ dernières années. D'un autre côté, souvent une petite entreprise ne pourra soumissionner sur de tels contrats (ex : UQAM, Biodôme) car elle est incapable d'avancer les fonds nécessaires à la mise en place du système de sécurité avant d'être payée par le client.

En faisant le suivi des entreprises montréalaises tentant de s'implanter sur le marché des services de gardiennage privé, sur une période de dix ans, on a calculé que leur espérance de vie était de quatre ans. Ce laps de temps est trop court pour que l'agence se bâtisse une réputation. On en conclut qu'il existe bel et bien des barrières à l'entrée que beaucoup d'entrepreneurs 
ne voient pas, ce qui les amène à essayer malgré tout d'investir le marché. Ils devront rapidement, pour la plupart, mettre la clé sous le paillasson'.

\section{Réputation}

Étant donné qu'a priori le client ne connaît pas la qualité du bien qu'il va acheter ou du service dont il va se doter, il se base sur un certain nombre d'indicateurs afin de fixer son choix sur le fournisseur de bien ou de services avec lequel il fera affaire. À cet effet, la réputation de l'entreprise est par conséquent importante. Par réputation, il faut entendre non seulement le nom de l'entreprise, mais aussi son expérience, ses années d'existence...

Dans le cadre de notre étude, afin de quantifier la réputation des entreprises de sécurité privée sur le marché, les années d'existence des agences ont été prises en compte, l'hypothèse étant que plus une entreprise est vieille, plus elle aura eu le temps de faire connaitre son nom et de se tailler une réputation.

Se basant sur la date de constitution des entreprises ${ }^{10}$, on constate (graphique 3) que la plus vieille entreprise présente sur le marché québécois de la sécurité privée n'a que trente ans ${ }^{\text {"I }}$. On constate aussi que le nombre d'entreprises montréalaises est distribué de façon quasi uniforme en fonction de l'ancienneté, alors qu'en dehors de Montréal, beaucoup d'entreprises ont été créées à partir de 1990 . Cela indique que les agences hors Montréal sont plus jeunes que celles de Montréal.

En mettant en relation la taille des entreprises et leur ancienneté, on devrait trouver que plus une entreprise est ancienne, plus elle compte d'agents, car plus son existence est connue. Nous avons tenté d'établir cette relation, retirant pour ce faire quelques entreprises de la population : celles n'ayant pas déclaré le nombre de leurs agents et les quatre entreprises employant plus de 1000 agents. La raison de ce dernier retrait est la trop grande importance qu'auraient prise ces entreprises dans la régression puisqu'il s'agit de valeurs extrêmes.

9. Bien que, dans certains cas, il s'agisse d'entreprises fantômes. En effet, certaines agences sont créées dans le seul but de décrocher des contrats, mais elles n'ont pas les ressources nécessaires pour les honorer. Par conséquent, elles revendent des franchises ou se font racheter. Une telle pratique est illégale.

10. Les dates de constitution des entreprises sont tirées des dossiers ouverts au Bureau d'enregistrement des raisons sociales. Quelques corrections ont dû être apportées. On en trouve le détail dans le mémoire de Degailler (1997).

11. Il s'agit de Burns. Certaines entreprises travaillent à la fois dans et hors Montréal. Cinq entreprises ont été formées après une fusion : Garda (1986), Barnes (1991), Protectron (1991), Bradson Mercantile (1991) et Service de sécurité de l'Est (1992). Ce qui signifie qu'en fait ces entreprises sont plus anciennes que la date officielle de leur fondation ne le laisse penser et que leur nom était connu avant l'année indiquée entre parenthèses. 


\section{Graphique 3}

Nombre d'entreprises en relation avec les années d'ancienneté (1996)

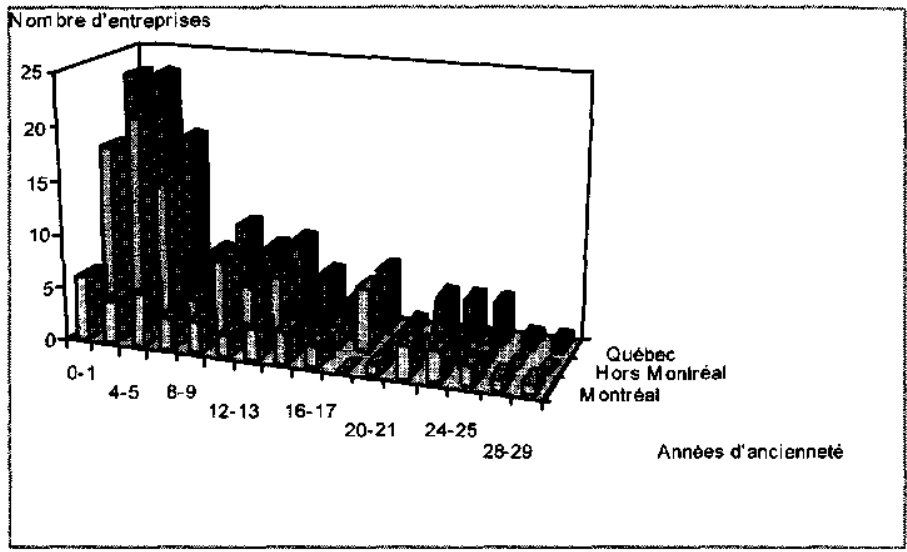

Tableau 2

Taille des entreprises de Montréal en fonction de leur ancienneté

\begin{tabular}{|c|c|c|c|}
\hline Variable & Constante & Années d'existence & R-carré \\
\hline $\begin{array}{c}\text { Nombre d'employés } \\
\text { (Taille) }\end{array}$ & $\begin{array}{c}15,11 \\
(0,29)\end{array}$ & $\begin{array}{c}9,31 \\
(2,77)^{*}\end{array}$ & 0,19 \\
\hline
\end{tabular}

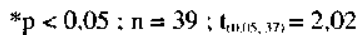

Le premier nombre est la valeur du coefficient, le nombre entre parenthèses est la valeur du t-stat.

Tableau 3

Taille des entreprises hors Montréal en fonction de leur ancienneté

\begin{tabular}{|c|c|c|c|}
\hline Variable & Constante & Années d'existence & R-carré \\
\hline $\begin{array}{c}\text { Nombre d'employés } \\
\text { (Taille) }\end{array}$ & 10,11 & 5,27 & 0.22 \\
\hline
\end{tabular}

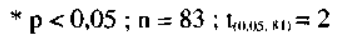

Le premier nombre est la valeur du coefficient, le nombre entre parenthèses est la valeur du $\mathrm{t}$-stat. 
Tableau 4

Taille des entreprises en fonction de leur ancienneté pour le Québec

\begin{tabular}{|c|c|c|c|}
\hline Variable & Constante & Années d'existence & R-carré \\
\hline $\begin{array}{c}\text { Nombre d'employés } \\
\text { (Taille) }\end{array}$ & $\begin{array}{c}1,42 \\
(0,09)\end{array}$ & $\begin{array}{c}7,83 \\
(5,73)^{*}\end{array}$ & 0,23 \\
\hline
\end{tabular}

$*_{p}<<0,05 ; n=115 ; t_{0.010 .11 .3}=1,98$

Le premier nombre est la valeur du coefficient, le nombre entre parenthèses est la valeur du t-stat.

La variable expliquée est la taille de l'entreprise (représentée par le nombre d'agents employés) et la variable explicative son ancienneté (nombre d'années d'existence).

Dans les trois cas de figure - Montréal, hors Montréal et province de Québec - la relation entre la taille de l'entreprise et son ancienneté apparaît significative. Cependant, à chaque fois la variance expliquée est faible $(19 \%, 22 \%$ et $23 \%)$. Le graphique 4 (relation entre l'ancienneté et le nombre d'employés) permet de mieux distinguer les entreprises ayant un comportement spécial, chaque point correspondant à une entreprise. La première colonne correspond à zéro employé, la deuxième : 1 à 5 employés, puis 6 à 10,11 à 25,26 à 49,50 à 99 et 100 à 249 employés.

\section{Graphique 4}

Relation taille-ancienneté pour les entreprises de sécurité à contrat en fonction au Québec en 1996

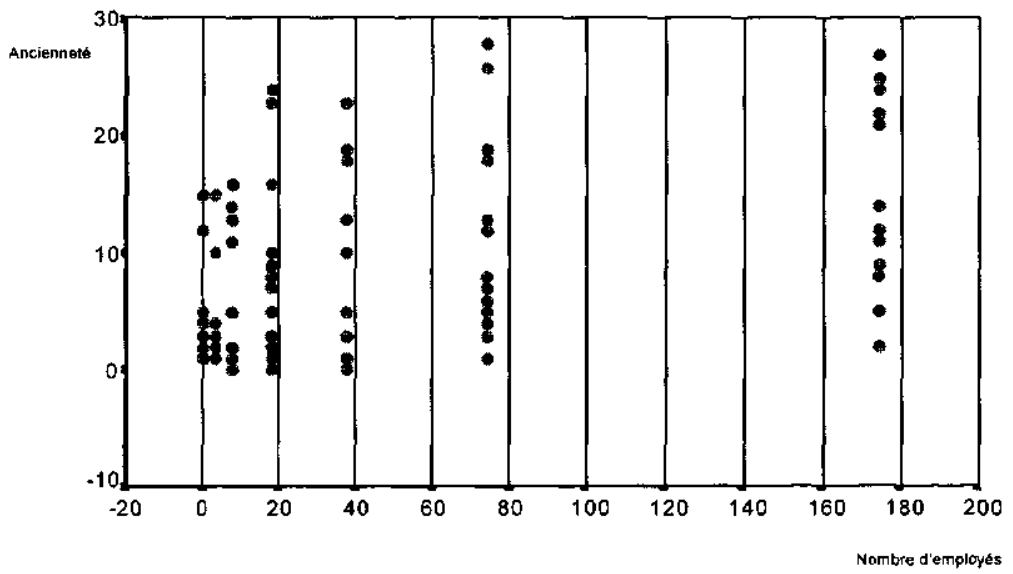


On constate l'existence de deux catégories d'outsiders: la première correspond aux entreprises jeunes (moins de 5 ans d'existence) de taille moyenne (supérieure à 60 employés). Trois explications au moins sont à ce sujet possibles : 1) la date de fondation qui nous est connue est erronée ; 2) l'entreprise est déjà grande lorsqu'elle s'implante sur le marché ${ }^{12}$;3) l'entreprise s'est installée dans une région où elle n'a pas affaire à beaucoup de concurrence.

La deuxième catégorie d'outsiders concerne des entreprises présentes depuis longtemps sur le marché, mais qui sont demeurées petites (plus de 10 ans d'existence, moins de 100 employés). Ici, cinq explications sont possibles : 1) le nombre d'employés rapporté est faux ; 2) ces entreprises n'ont pas pu grandir à cause de l'existence de certaines barrières ; 3) ces entreprises possèdent une niche ; 4) la sécurité n'est pas leur domaine d'activité principal ; 5) elles sont en voie de faire faillite.

Vingt-six entreprises entrent dans cette dernière catégorie d'outsiders. Parmi ces 26 entreprises, sept possèdent une niche de spécialisation par rapport à leurs activités en sécurité privée (escorte des entrepreneurs sur les pistes d'atterrissage des aéroports, vente d'équipements de protection individuelle et de lutte contre l'incendie, service de recouvrement, service de maintien à domicile, autres services personnels et domestiques, hygiène de l'environnement...). Quant aux 14 entreprises restantes, neuf occupent une niche situationnelle. C'est-à-dire que dans la région dans laquelle elles se trouvent et opèrent ${ }^{13}$, le nombre d'agences fournissant des services de sécurité est restreint (elles y sont souvent les seules). La niche situationnelle est moins stable que la niche de spécialisation, car une grande entreprise peut toujours venir s'installer dans la région et drainer la clientèle, alors qu'il faudrait être armé pour se lancer dans la fourniture de services spécialisés normalement offerts par une entreprise déjà bien équipée pour faire face à la demande et souvent bien ancrée sur le marché demandant spécifiquement de tels services.

En regard des considérations précédemment énoncées, la situation de concurrence parfaite en ce qui a trait à la fourniture de services de gardiennage privé, au Québec, peut définitivement être éliminée au profit de l'oligopole avec niche. Un autre cas de figure peut encore être envisagé, celui de l'oligopole avec leader, situation voulant qu'il existe une entreprise

12. Il s'agit d'entreprises déjà implantées ailleurs ou ayant d'autres secteurs d'activités leur permettant d'avancer suffisamment de fonds pour ouvrir une nouvelle entreprise de taille raisonnable.

13. La région peut soit être un district soit un endroit localisé (ex : un quartier). 
dominante fixant les règles du jeu, les autres entreprises observant son comportement ou l'anticipant afin de déterminer leur propre politique d'activité.

Il existe, à l'heure actuelle, au Québec, plusieurs entreprises qui se démarquent. Parmi celles-ci : Sécurité et protection Secpro, Sécurité Kolossal Inc, Pinkerton du Québec Limitée, le Groupe de sécurité Garda et Burns.

Visiblement, 1996 a été une année clé permettant de savoir si une agence deviendrait dominante. On y assiste à une phase de concentration importante du marché québécois de la sécurité privée, de sorte qu'à l'heure actuelle celuici se dessinerait de la manière suivante : «Parmi les 219 agences de sécurité (4 $^{4}$ au Québec, une dizaine réaliserait $80 \%$ des revenus de l'industrie... L'acquisition de Sélect par Pinkerton crée une agence dominante sur le marché québécois. Elle sera presque deux fois plus grosse que ses plus proches rivales de taille moyenne. »

Bien que, sur plusieurs points, le marché des agents de sécurité observé au Québec s'apparente à une concurrence parfaite (nombre d'entreprises, homogénéité du prix et quasi homogénéitê du produit), on doit plutôt conclure que le marché des agents de sécurité est un oligopole et que Pinkerton essaie d'en devenir le leader (avec plus de 3000 agents).

Le seul fait que l'on soit dans une situation d'oligopole de type leader indique, par ailleurs, que le marché n'est plus en croissance.

Ce fait se confirme lorsqu'on examine le nombre d'heures travaillées par les agents de sécurité au cours des années, lequel témoigne d'une baisse de la demande).

\section{Baisse de la demande}

Comme le prix des services ne fluctue presque pas, afin d'estimer la demande, les données transmises par le Comité paritaire concernant le nombre d'heures travaillées par les agents de sécurité de 1989 à 1995 peuvent servir de base d'estimation de la quantité de bien ou de service demandée, et donc de la demande (en fait il s'agit de pointes d'équilibre successives entre l'offre et la demande).

Tableau 5

Nombre d'heures travaillées au Québec de 1989 à 1995

\begin{tabular}{|c|c|c|c|c|c|c|c|}
\hline & 1989 & 1990 & 1991 & 1992 & 1993 & 1994 & 1995 \\
\hline Nb heures & 23408174 & 23003048 & 23018045 & 22762661 & 21654067 & 20218906 & 19306182 \\
\hline
\end{tabular}

14. En fait, il s'agit de 219 agences de sécurité et d'investigation. 
On constate que le nombre d'heures travaillées par les agents de sécurité au Québec diminue continuellement depuis 1989. Afin de vérifier si cette baisse est significative, on teste l'hypothèse unilatérale $\mathrm{H}_{\mathrm{A}}$ : $\mathrm{b}<0$ contre $\mathrm{H}_{0}$ : $\mathrm{b}=0, \mathrm{~b}$ étant le coefficient de croissance. Le seuil critique de la statistique de Student à $5 \%$ pour 7 observations est $t_{(0.05 .5)}=-2,02$.

\section{Tableau 6}

Vérification de la tendance pour le nombre d'heures travaillées au Québec (1989-1995)

\begin{tabular}{|c|c|c|c|}
\hline Variable & Constante & Tendance & R-carré \\
\hline $\begin{array}{c}\text { Nombre d'heures } \\
\text { travaillées }\end{array}$ & $\begin{array}{c}21910231,8 \\
(94,53)\end{array}$ & $\begin{array}{c}-6871137,83 \\
(-5,929)^{*}\end{array}$ & 0,88 \\
\hline
\end{tabular}

$* \mathrm{p}<0,05$

Le premier nombre est la valeur du coefficient. le nombre entre parenthèses est la valeur du t-stat.

La baisse apparaît fortement significative. On sait cependant que le nombre d'heures travaillées par les agents de sécurité est sous-estimé car les entreprises ne déclarent pas certaines heures supplémentaires et parfois même certains travailleurs au noir. Mais, même en considérant que les entreprises ne trichaient pas en 1989 et qu'elles le font maintenant, pour que la baisse observée du nombre d'heures travaillées ne soit pas réelle, il faudrait que les entreprises trichent d'environ $20 \%$ sur les données qu'elles transmettent actuellement au Comité, ce qui paraît peu réaliste.

\section{Graphique 5}

Nombre d'heures travaillées par les agents de sécurité privée au Québec de 1989 à 1995

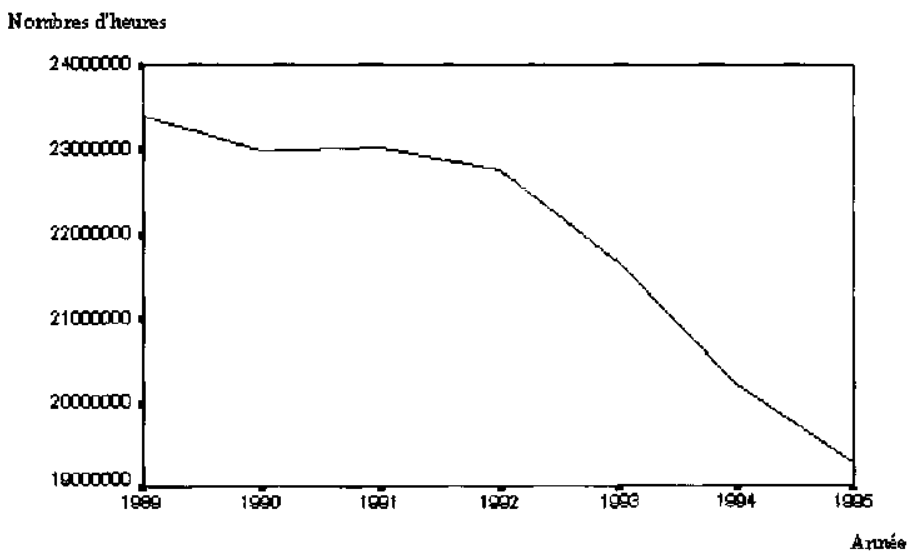


Le graphique 5 montre que 1992 est une année charnière à partir de laquelle le nombre d'heures travaillées descend rapidement, alors que la série était relativement plane jusque-là. Afin de vérifier si l'on est en présence d'une brisure, on régresse le nombre d'heures travaillées sur une constante, une tendance et une tendance cassée ${ }^{15}$ (tableau 7 ).

\section{Tableau 7}

Vérification de la brisure quant au nombre d'heures travaillées par les agents de sécurité à contrat au Québec (1989-1995)

\begin{tabular}{|c|c|c|c|c|}
\hline Variable & Constante & Tendance & Tendance cassée & R-carré \\
\hline & & & & \\
Nombre d'heures & 22755326,1 & $-194166,15$ & $-985943,37$ & 0,99 \\
travaillées & $(226,27)$ & $(-3,43)^{*}$ & $(-9,81)^{*}$ & \\
\hline
\end{tabular}

* $\mathrm{p}<0.05, \mathrm{n}=7, \mathrm{k}_{\mathrm{n}(\mathrm{n}, 15,3)}=-2.02$

Le premier nombre est la valeur du coefficient, le nombre entre parenthèses est la valeur du t-stat.

Il y a effectivement une brisure en 1992. La demande commence à baisser dès 1990, mais elle a subi un choc en 1992, dont elle ne se relèvera pas par la suite, en tout cas aussi loin que nous puissions suivre la série. En fait, rien ne nous permet d'affirmer que cette tendance va se poursuivre dans l'avenir car elle peut être la résultante de la crise économique vécue au Québec. Si une reprise survient, ce secteur d'activité peut fort bien repartir.

\section{CONCLUSION}

Trois considérations seront rapidement amenées en conclusion. D'abord il est particulièrement malaisé de se prononcer de manière catégorique quant à l'évolution du marché de la sécurité privée, du moins au Québec, actuellement. Nous avons pu montrer que la seule étude de l'évolution du nombre d'agences et du nombre d'agents de sécurité privée, comme indicateurs de cette évolution, entraîne de si nombreux biais qu'elle ne peut être prise qu'à titre indicatif, justement. Une étude de marché en tant que telle, comme les économistes la conçoivent et la pratiquent, se révélerait sans contredit plus juste. Mais la possibilité même đ'une telle étude souffre actuellement du manque flagrant de données concernant le secteur d'activité de la sécurité privée et ses champs d'application. D'une part, les comptes que doivent rendre les agences de sécurité privée sont peu encadrés et somme toute assez sommaires; d'autre part, les agences de sécurité privée ne sont pas

15. Les valeurs de cette tendance sont zéro jusqu'en 1992, puis valent $1,2,3$. 
particulièrement enclines à rendre des comptes qui ne leur sont pas demandés. Enfin, tout un pan de la sécurité privée, lié aux technologies de surveillance, demeure totalement occulte.

De sorte que si, malgré toutes les difficultés que nous avons rencontrées et toutes les précautions que nous avons prises, nous avons pu établir que les activités de gardiennage privé pratiquées au Québec régressaient à l'heure actuelle, et que les activités d'investigation privée y étaient encore peu développées, nous ne pouvons nullement conclure à une diminution des activités de contrôle relevant du secteur privé. Rien ne permet, en effet, de dire que celles-ci ne s'étendent pas, au contraire, de manière tentaculaire et de plus en plus pernicieuse parce que de moins en moins visible (la miniaturisation des instruments technologiques s'étant depuis longtemps réalisée), étayant ainsi l'inquiétude de Marx et de ses adeptes. Mais rien ne permet d'affirmer que tel est effectivement le cas, ce qui laisse une part d'espoir à Clarke et à ceux qui, comme lui, prétendent que l'humain ne se laissera pas prendre par l'œil magique de la technologie.

\section{RÉFÉRENCES}

BRODEUR, J.-P. (1995), « Le contrôle social : privatisation et technocratie », Déviance et société, vol. 19, $\mathrm{n}^{\circ} 2$, p. 127-147.

CLARKE, R. V. (1995), «Les technologies de la prévention situationnelle », Les Cahiers de la sécurité intérieure, vol. 21, p. 101-113.

COUSINEAU, M.-M. et NORMANDEAU, A. (1993), Les cô̂ts sociaux et économiques de la criminalité, Montréal, Université de Montréal, École de criminologie.

CUNNINGHAM, W., STAUSCHS, J. J, et VAN METER, C. (1990), The Hallcrest report II : Private Security Trends 1970-2000, Boston, Butterworth-Heinemann.

CUSSON, M. (1992), "L'analyse criminologique de la prévention situationnelle », Revue internationale de criminologie et de police technique, vol. 45, $\mathrm{n}^{\circ} 2$, p. 137-149.

DEDECKER, R. (1991), La sécurité privée dans l'Europe des Douze, Bruges, Vanden Broele.

DEGAILLER, F. (1997), Étude de marché des agences de sécurité à sontrat à Montréal et à Québec (1986-1996), Montréal, École de criminologie, Université de Montréal, mémoire de maîtrise inédit.

FARNELl, M. et SHEARING, C. (1977), Private security : an examination of canadian statistics, 196/.1971, Toronto, Centre of Criminology, University of Toronto.

FOURCAUDOT, M. (1988), Étude descriptive sur les agences de sécurité privée au Québec, Montréal, Université de Montréal, École de criminologie, mémoire de maîtrise inédit.

LIPMAN, M. et McGRAW, W. (1988), "Employee theft : A 40 \$ billion industry », The Annals of the American Academy, vol. 498, juillet, p. 51-59.

McCRIE, R. (1988), «The development of the U.S. security industry », The Annals of the American Academy, vol. 498, juillet, p. 23-33.

MARX, G. T. (1988), «La Société de Sécurité Maximale », Déviance et société, vol. 12, $n^{\circ} 2$, p. 147-166. 
NOGALA, N. (1996), «Le marché de la sécurité privée : analyse d'une évolution internationale ", Les Cahiers de la sécurité intérieure, vol. 24, p. 121-141.

OCQUETEAU, F. (1986), «Police(s) privée(s), sécurité privée : nouveaux enjeux de l'ordre et du contrôle social », Déviance et société, vol, 10, n 3, p. 247-281.

OCQUETEAU, F. (1992), Gardiennage, surveillance et sécurité privée : commerce de la peur et/ou peur du commerce?, Paris, CESSDIP.

OCQUETEAU, F. (1994), "La sécurité "marchandisée" », Projet : insécurité : question de confiance, vol. 238, p. 63-72.

SHEARING, C. (1984), « La sécurité privée au Canada : Quelques questions et réponses 》, Criminologie, vol. 17, p. 59-89.

SHEARING, C. et STENNING, P. (1983), «Private Security : implication for social control », Social Problems, vol. 30, n $\mathrm{n}^{\circ}$ 5, p. 493-506.

SHEARING, C. et STENNING P. (1987), «Reframing Policing » in SHEARING C. et STENNING P. (dir.), Privale Policing, (vol. 23, p. 9-18), Sage, Criminal Justice System Annuals.

SPITZER, S. et SCULL, A. (1977), « Social control in historical perspective : from private to public response to crime ", Corrections and punishment, vol. 8, p. 265-285.

TRUETT, R., TILLET, B. et VAN METER, C. (1988), " Principles of security ", Criminal Justice Studies, Anderson Publishing co., deuxième édition. 